PROCEEDINGS OF THE AMERICAN MATHEMATICAL SOCIETY

Volume 124, Number 11, November 1996

\title{
TOEPLITZ OPERATORS ON THE POLYDISK
}

\author{
SUNHUA SUN AND DECHAO ZHENG
}

(Communicated by Palle E. T. Jorgensen)

\begin{abstract}
In this paper it is shown that two analytic Toeplitz operators essentially doubly commute if and only if they doubly commute on the Bergman space of the polydisk.
\end{abstract}

Let $D$ be the open unit disk in $C$. Its boundary is the circle $T$. The polydisk $D^{n}$ and the torus $T^{n}$ are the subsets of $C^{n}$ which are cartesian products of $n$ copies $D$ and $T$, respectively. Let $d A(z)$ be the normalized volume measure on $D^{n}$. The Bergman space $L_{a}^{2}\left(D^{n}\right)$ is the subspace of $L^{2}\left(D^{n}, d A\right)$ whose functions are holomorphic in $D^{n}$. There is an orthogonal projection $P$ from $L^{2}\left(D^{n}, d A\right)$ onto $L_{a}^{2}\left(D^{n}\right)$. The Toeplitz operator with symbol $f$ in $L^{\infty}\left(D^{n}\right)$ is defined by $T_{f}(h)=$ $P(f h)$, for all $h \in L_{a}^{2}\left(D^{n}\right)$. Two analytic Toeplitz operators $T_{f}$ and $T_{g}$ are said to be essentially doubly commuting if $T_{f}^{*} T_{g}-T_{g} T_{f}^{*}$ is compact. They are said to be doubly commuting if $T_{f}^{*} T_{g}-T_{g} T_{f}^{*}=0$.

The function theory on the polydisk $D^{n}$ is quite different from the function theory on the unit disk $[R]$. One may expect that there should exist some differences in operator theory on the Bergman spaces between on the polydisk and on the disk. In this paper we will show that two analytic Toeplitz operators essentially doubly commute if and only if they doubly commute on the polydisk. But this is false on the disk $[\mathrm{AG}],[\mathrm{Z}]$.

Observe that $T^{n}$ is only a small part of the boundary of $D^{n}$ if $n>1$. But it is an important part and is called the distinguished boundary of $D^{n}$. $T^{n}$ is also a compact group (with componentwise multiplication as group operation) and as such carries a Haar measure. Its dual group is $Z^{n}$ where $Z$ is the integer group. As in $[\mathrm{SW}]$ we consider multiple Fourier series on the $n$-torus $T^{n}$. The multiple Fourier series on the $n$-torus $T^{n}$ can be viewed as the Fourier transformation on $L^{1}\left(T^{n}\right)$. For $f$ in $L^{1}\left(T^{n}\right)$ the Fourier transformation is given by

$$
\tilde{f}(\vec{m})=\int_{T^{n}} f\left(x_{1}, \ldots, x_{n}\right) e^{i(\vec{m}, \vec{x})} d \sigma\left(x_{1}\right) \cdots d \sigma\left(x_{n}\right)
$$

where $\vec{m}=\left(m_{1}, \ldots, m_{n}\right) \in Z^{n}$ and $(\vec{m}, \vec{x})=\sum_{i=1}^{n} m_{i} x_{i}$ and $\sigma_{i}\left(x_{i}\right)$ is the normalized Haar measure on $T$ for $i=1, \ldots, n$. By Theorem 1.7 in [SW], the Fourier transformation is injective, i.e. if $f \in L^{1}\left(T^{n}\right)$ and $\tilde{f}(m)=0$ for all $m \in Z^{n}$, then $f \equiv 0$. The main result in this paper is the following theorem.

Received by the editors October 6, 1994 and, in revised form, April 21, 1995.

1991 Mathematics Subject Classification. Primary 47B35.

Key words and phrases. Toeplitz operator, Bergman space, polydisk.

The first author was supported in part by the National Natural Science Foundation of China.

The second author was supported in part by the National Science Foundation. 
Theorem. Let $f$ and $g$ be two bounded analytic functions on the polydisk $D^{n}$ for $n>1$. Then the following are equivalent:

(A) $T_{f}$ essentially doubly commutes with $T_{g}$.

(B) $T_{f}$ doubly commutes with $T_{g}$.

(C) Either $\frac{\partial f}{\partial z_{i}}$ or $\frac{\partial g}{\partial z_{i}}$ is zero for all $i$.

Before beginning the proof of the Theorem, we need some notations and definitions. Since for any $z$ in $D^{n}$, the pointwise evaluation of functions in $L_{a}^{2}\left(D^{n}\right)$ at $z$ is a bounded functional, there is a function $K_{z}$ in $L_{a}^{2}\left(B_{n}\right)$ such that

$$
f(z)=\left(f, K_{z}\right)
$$

for all $f$ in $L_{a}^{2}\left(D^{n}\right) . K_{z}$ is called the Bergman reproducing kernel and sometimes we use $K(z, w)$ to denote $K_{z}(w)$.

Let $K_{z_{1}}$ denote the Bergman producing kernel $\frac{1}{\left(1-z_{1} w_{1}\right)^{2}}$ of the Bergman space $L_{a}^{2}(D)$ of the unit disk at the point $z_{1}$ in $D$, and $k_{z_{1}}$ the normalized Bergman reproducing kernel of the Bergman space $L_{a}^{2}(D)$ at the point $z_{1} \in D$. We use $z$ to denote the vector $\left(z_{1}, \ldots, z_{n}\right)$ in $C^{n}$ of an $n$-dimensional complex plane. It is easy to check that the reproducing kernel $K_{z}$ of the Bergman space $L_{a}^{2}\left(D^{n}\right)$ of the polydisk is the multiple product $\prod_{i=1}^{n} k_{z_{i}}\left(w_{i}\right)$ of the Bergman kernel of the unit disk $D$. So the normalized producing kernel $k_{z}$ of $L_{a}^{2}\left(D^{n}\right)$ is also the multiple product $\prod_{i=1}^{n} k_{z_{i}}$ of the normalized kernel of the unit disk. Then $k_{z}$ weakly converges to zero in $L_{a}^{2}\left(D^{n}\right)$ as $z$ tends to the boundary of $D^{n}$.

In addition, the producing kernel $K_{z}$ has the following nice property:

$$
T_{\bar{f}} K_{z}=\overline{f(z)} K_{z}
$$

for $z$ in $D^{n}$ if $f$ is in $L_{a}^{2}\left(D^{n}\right)$.

Let $Z_{+}^{n}$ denote the subset $\left\{\vec{m} \in Z^{n}: m_{i} \geq 0 \forall i=1, \ldots, n\right\}$ of $Z^{n}$. For a function $f$ in the Hardy space $H^{2}\left(T^{n}\right)$, we write the power series of $f$ as follows:

$$
f(z)=\sum_{\vec{m} \in Z_{+}^{n}} a_{\vec{m}} z^{\vec{m}}
$$

where $a_{\vec{m}}$ is a sequence of numbers such that

$$
\sum_{\vec{m} \in Z_{+}^{n}}\left|a_{\vec{m}}\right|^{2}<\infty
$$

and $z^{\vec{m}}$ means the product $\prod_{i=1}^{n} z_{i}^{m_{i}}[\mathrm{R}]$.

Proof of the Theorem. Obviously $(\mathrm{B}) \Rightarrow(\mathrm{A})$. First we prove that $(\mathrm{C}) \Rightarrow(\mathrm{B})$. Without loss of generality we assume that $f(z)=F\left(z_{1}, \ldots, z_{r}\right)$ and $g(z)=G\left(z_{r+1}, \ldots, z_{n}\right)$. Since the Bergman kernel of $D^{n}$ is the multiple product $\prod_{i=1}^{n} K_{z_{i}}\left(w_{i}\right)$ of the Bergman kernel of $D$, by (1), we have

$$
\left(T_{f} T_{g}^{*} \prod_{i=1}^{n} K_{z_{i}}\right)(w)=F\left(w_{1}, \ldots, w_{r}\right) \bar{G}\left(z_{r+1}, \ldots, z_{n}\right) \prod_{i=1}^{n} K_{z_{i}}\left(w_{i}\right)
$$


and

$$
\begin{aligned}
\left(T_{g}^{*} T_{f} \prod_{i=1}^{n} K_{z_{i}}\right)(w) & =F\left(w_{1}, \ldots, w_{r}\right) \prod_{i=1}^{r} K_{z_{i}}\left(w_{i}\right)\left(T_{g}^{*} \prod_{i=r+1}^{n} K_{z_{i}}\right)\left(w_{r+1}, \ldots, w_{n}\right) \\
& =F\left(w_{1}, \ldots, w_{r}\right) \bar{G}\left(z_{r+1}, \ldots, z_{n}\right) \prod_{i=1}^{n} K_{z_{i}}\left(w_{i}\right) .
\end{aligned}
$$

Thus

$$
T_{f} T_{g}^{*}\left(\prod_{i=1}^{n} K_{z_{i}}\right)=T_{g}^{*} T_{f}\left(\prod_{i=1}^{n} K_{z_{i}}\right) .
$$

This implies that $T_{f} T_{g}^{*}=T_{g}^{*} T_{f}$ since the values of an operator on the Bergman kernels completely determine the operator on the Bergman space.

$(\mathrm{A}) \Rightarrow(\mathrm{C})$ Since $D^{n}$ is symmetric with respect to all $z_{i}$, it is sufficient to prove that either $\frac{\partial f}{\partial z_{1}}$ or $\frac{\partial g}{\partial z_{1}}$ is zero. By (1), the elementary computation gives

$$
\begin{aligned}
& \left(\left(T_{f} T_{g}^{*}-T_{g}^{*} T_{f}\right) k_{z}, k_{z}\right) \\
& \quad=\int_{D^{n}} f(w) \bar{g}(w)\left|\prod_{i=1}^{n} k_{z_{i}}\left(w_{i}\right)\right|^{2} d A(w)-f(z) \bar{g}(z)
\end{aligned}
$$

for any $z$ in $D^{n}$. Let $\vec{\theta}=\left(\theta_{1}, \ldots, \theta_{n}\right)$ and $U_{\vec{\theta}}=\operatorname{diag}\left\{e^{i \theta_{1}}, \ldots, e^{i \theta_{n}}\right\}$. Replacing $z$ by $U_{\vec{\theta}} z$ in (2) yields

$$
\begin{aligned}
& \left(\left(T_{f} T_{g}^{*}-T_{g}^{*} T_{f}\right) k_{U_{\vec{\theta}}}, k_{U_{\vec{\theta}} z}\right) \\
& \quad=\int_{D^{n}} f(w) \bar{g}(w)\left|\prod_{j=1}^{n} k_{e^{i \theta_{j}} z_{j}}\left(w_{j}\right)\right|^{2} d A(w)-f\left(U_{\vec{\theta}} z\right) \bar{g}\left(U_{\vec{\theta}} z\right) .
\end{aligned}
$$

Multiplying (3) by $e^{i(\vec{m}, \vec{\theta})}$ and then integrating with respect to $\vec{\theta}$ imply

$$
\begin{aligned}
\int_{T^{n}}( & \left.\left(T_{f} T_{g}^{*}-T_{g}^{*} T_{f}\right) k_{U_{\vec{\theta}} z}, k_{U_{\vec{\theta}} z}\right) e^{i(\vec{m}, \vec{\theta})} d \theta \\
= & \int_{D^{n}} \int_{T^{n}} f\left(U_{\vec{\theta}} w\right) \bar{g}\left(U_{\vec{\theta}} w\right)\left|\prod_{j=1}^{n} k_{z_{j}}\left(w_{j}\right)\right|^{2} e^{i(\vec{m}, \vec{\theta})} d A(w) d \theta \\
& -\int_{T^{n}} f\left(U_{\vec{\theta}} z\right) \bar{g}\left(U_{\vec{\theta}} z\right) e^{i(\vec{m}, \vec{\theta})} d \theta .
\end{aligned}
$$

Let $H_{\vec{m}}(z)=\int_{T^{n}} f\left(U_{\vec{\theta}} z\right) \bar{g}\left(U_{\vec{\theta}} z\right) e^{i(\vec{m}, \vec{\theta})} d \theta$. Since both $f$ and $g$ are in $H^{\infty}\left(T^{n}\right)$, we write

$$
f=\sum_{\vec{l} \in Z_{+}^{n}} a_{\vec{l}} z^{\vec{l}} \text { and } g=\sum_{\vec{j} \in Z_{+}^{n}} b_{\vec{j}} z^{\vec{j}}
$$

where $a_{\vec{l}}$ and $b_{\vec{j}}$ are two sequences of numbers such that

$$
\sum_{\vec{l} \in Z_{+}^{n}}\left|a_{\vec{l}}\right|^{2}<\infty \text { and } \sum_{\vec{j} \in Z_{+}^{n}}\left|b_{\vec{j}}\right|^{2}<\infty .
$$


To show that $H_{\vec{m}}(z)$ is continuous on the closure $\bar{D}^{n}$ of the polydisk, for the sake of convenience, we consider the special case that $\vec{m} \in Z_{+}^{n}$. Then

$$
H_{\vec{m}}(z)=\sum_{\vec{l} \in Z_{+}^{n}} a_{\vec{l}} \overline{b_{(\vec{l}+\vec{m})}} z^{\vec{l}} \bar{z}^{(\vec{l}+\vec{m})} .
$$

Since

$$
\sum_{\vec{l} \in Z_{+}^{n}}\left|a_{\vec{l}} \overline{b_{(\vec{l}+\vec{m})}}\right| \leq\left(\sum_{\vec{l} \in Z_{+}^{n}}\left|a_{\vec{l}}\right|^{2}\right)^{1 / 2}\left(\sum_{\vec{l} \in Z_{+}^{n}}\left|b_{\vec{l}}\right|^{2}\right)^{1 / 2}<\infty
$$

$H_{\vec{m}}(z)$ is continuous on the closure $\bar{D}^{n}$ of $D^{n}$ for any $\vec{m} \in Z^{n}$. It follows from (4) that

$$
\begin{aligned}
& \int_{T^{n}}\left(\left(T_{f} T_{g}^{*}-T_{g}^{*} T_{f}\right) k_{U_{\vec{\theta}}}, k_{U_{\vec{\theta}}}\right) e^{i(\vec{m}, \vec{\theta})} d \theta \\
& \quad=\int_{D^{n}} H_{\vec{m}}(w)\left|k_{z}(w)\right|^{2} d A(w)-H_{\vec{m}}(z) .
\end{aligned}
$$

Let $\phi_{z_{i}}\left(w_{i}\right)$ be the Möbius map $\frac{z_{i}-w_{i}}{1-z_{i} w_{i}}$. Changing variables $w_{i}=\phi_{z_{i}}\left(\lambda_{i}\right)$ for $i=$ $2, \ldots, n$ in (5), we have

$$
\begin{aligned}
\int_{T^{n}} & \left(\left(T_{f} T_{g}^{*}-T_{g}^{*} T_{f}\right) k_{U_{\vec{\theta}}}, k_{U_{\vec{\theta}} z}\right) e^{i(\vec{m}, \vec{\theta})} d \theta \\
= & \int_{D^{n}} H_{\vec{m}}\left(w_{1}, \phi_{z_{2}}\left(\lambda_{2}\right), \ldots, \phi_{z_{n}}\left(\lambda_{n}\right)\right)\left|k_{z_{1}}\right|^{2} d A\left(w_{1}\right) d A\left(\lambda_{2}\right) \cdots d A\left(\lambda_{n}\right) \\
& -H_{\vec{m}}\left(z_{1}, \ldots, z_{n}\right) .
\end{aligned}
$$

Let $\left(\mu_{2}, \ldots, \mu_{n}\right)$ be a point in $T^{n-1}$. Since $n>1$, we can choose a sequence $\left\{\left(z_{2}, \ldots, z_{n}\right)_{\alpha}\right\} \subset D^{n-1}$ converging to $\left\{\mu_{2}, \ldots, \mu_{n}\right\}$. For a fixed $z_{1} \in D$, let $z_{\alpha}=$ $\left(z_{1}, \ldots, z_{n}\right)_{\alpha}$. Since $k_{z}$ weakly converges to zero as $z$ goes to the boundary of $D^{n}$ and $T_{f} T_{g}^{*}-T_{g}^{*} T_{f}$ is compact,

$$
\lim _{(z)_{\alpha}} \int_{T^{n}}\left(\left(T_{f} T_{g}^{*}-T_{g}^{*} T_{f}\right) k_{U_{\vec{\theta}} z_{\alpha}}, k_{U_{\vec{\theta}} z_{\alpha}}\right) e^{i(\vec{m}, \vec{\theta})} d \theta=0 .
$$

Then we conclude that

$$
\begin{aligned}
& \lim _{z_{\alpha}} \int_{D^{n}} H_{\vec{m}}\left(w_{1}, \phi_{\left(z_{2}\right)_{\alpha}}\left(\lambda_{2}\right), \ldots, \phi_{\left(z_{n}\right)_{\alpha}}\left(\lambda_{n}\right)\right)\left|k_{z_{1}}\right|^{2} d A\left(w_{1}\right) d A\left(\lambda_{2}\right) \cdots d A\left(\lambda_{n}\right) \\
& \quad=H_{\vec{m}}\left(z_{1}, \mu_{2}, \ldots, \mu_{n}\right) .
\end{aligned}
$$

Because $H_{\vec{m}}\left(w_{1}, \ldots, w_{n}\right)$ is continuous on $\bar{D}^{n}$ and $\phi_{\left(z_{i}\right)_{\alpha}}\left(\lambda_{i}\right)$ converges to $\mu_{i}$ pointwise for $i=2, \ldots, n$, by the dominated convergence theorem, we thus have

$$
\int_{D} H_{\vec{m}}\left(w_{1}, \mu_{2}, \ldots, \mu_{n}\right)\left|k_{z_{1}}\right|^{2} d A\left(w_{1}\right)=H_{\vec{m}}\left(z_{1}, \mu_{2}, \ldots, \mu_{n}\right) .
$$

So $H_{\vec{m}}\left(z_{1}, \mu_{2}, \ldots, \mu_{n}\right)$ is continuous on $\bar{D}$ and satisfies the invariant volume mean value property, i.e.

$$
\int_{D} H_{\vec{m}}\left(\phi_{z_{1}}\left(w_{1}\right), \mu_{2}, \ldots, \mu_{n}\right) d A\left(w_{1}\right)=H_{\vec{m}}\left(z_{1}, \ldots, \mu_{2}, \ldots, \mu_{n}\right)
$$


for all $z_{1} \in D$. By [AFR], it is harmonic. Thus $\Delta_{z_{1}} H_{\vec{m}}\left(z_{1}, \mu_{2}, \ldots, \mu_{n}\right)=0$. On the other hand, if we write

$$
f\left(z_{1}, z_{2}, \ldots, z_{n}\right)=\sum_{k=0}^{\infty} \frac{\partial^{k} f}{\partial z_{1}^{k}}\left(0, z_{2}, \ldots, z_{n}\right) z_{1}^{k}
$$

and

$$
g\left(z_{1}, z_{2}, \ldots, z_{n}\right)=\sum_{k=0}^{\infty} \frac{\partial^{k} g}{\partial z_{1}^{k}}\left(0, z_{2}, \ldots, z_{n}\right) z_{1}^{k}
$$

and let $\vec{m}=\left(0, m_{2}, \ldots, m_{n}\right)$, then

$$
\begin{aligned}
H_{\vec{m}}\left(z_{1}, \mu_{2}, \ldots, \mu_{n}\right)=\sum_{k=0}^{\infty} \int_{T^{n-1}} & \frac{\partial^{k} f}{\partial z_{1}^{k}}\left(0, \mu_{2} e^{i \theta_{2}}, \ldots, \mu_{n} e^{i \theta_{n}}\right) \\
& \times \frac{\partial^{k} g}{\partial z_{1}^{k}}\left(0, \mu_{2} e^{i \theta_{2}}, \ldots, \mu_{n} e^{i \theta_{n}}\right) e^{i(\vec{m}, \vec{\theta})} d \theta_{2} \cdots d \theta_{n}\left|z_{1}\right|^{2 k},
\end{aligned}
$$

and

$$
\|f\|_{2}^{2}=\sum_{k=0}^{\infty} \int_{T^{n-1}}\left|\frac{\partial^{k} f}{\partial z_{1}^{k}}\left(0, e^{i \theta_{2}}, \ldots, e^{i \theta_{n}}\right)\right|^{2} d \theta_{2} \cdots d \theta_{n} .
$$

Hence we obtain

$$
\int_{T^{n-1}} \frac{\partial f}{\partial z_{1}}\left(0, \mu_{2} e^{i \theta_{2}}, \ldots, \mu_{n} e^{i \theta_{n}}\right) \overline{\frac{\partial g}{\partial z_{1}}\left(0, \mu_{2} e^{i \theta_{2}}, \ldots, \mu_{n} e^{i \theta_{n}}\right)} e^{i(\vec{m}, \vec{\theta})} d \theta_{2} \cdots d \theta_{n}=0
$$

for all $\vec{m} \in Z^{n-1}$, and both $\frac{\partial f}{\partial z_{1}}\left(0, z_{2}, \ldots, z_{n}\right)$ and $\frac{\partial g}{\partial z_{1}}\left(0, z_{2}, \ldots, z_{n}\right)$ are in $H^{2}\left(T^{n-1}\right)$. So $\frac{\partial f}{\partial z_{1}}\left(0, z_{2}, \ldots, z_{n}\right) \overline{\frac{\partial g}{\partial z_{1}}\left(0, z_{2}, \ldots, z_{n}\right)}$ is in $L^{1}\left(T^{n-1}\right)$ and the Fourier transformation of $\frac{\partial f}{\partial z_{1}}\left(0, z_{2}, \ldots, z_{n}\right) \overline{\frac{\partial g}{\partial z_{1}}\left(0, z_{2}, \ldots, z_{n}\right)}$ on $Z^{n-1}$ is zero. The injection of the Fourier transformation implies that $\frac{\partial f}{\partial z_{1}}\left(0, z_{2}, \ldots, z_{n}\right) \frac{\overline{\partial g}}{\partial z_{1}}\left(0, z_{2}, \ldots, z_{n}\right)$ is zero on $T^{n-1}$. Thus

$$
\frac{\partial f}{\partial z_{1}}\left(0, z_{2}, \ldots, z_{n}\right) \frac{\partial g}{\partial z_{1}}\left(0, z_{2}, \ldots, z_{n}\right)
$$

is zero on $T^{n-1}$.

Let $\phi_{z}(w)$ denote the Möbius transform $\left(\phi_{z_{1}}\left(w_{1}\right), \ldots, \phi_{z_{n}}\left(w_{n}\right)\right)$ in the polydisk $D^{n}$ for each point $z=\left(z_{1}, \ldots, z_{n}\right) \in D^{n}$. For a fixed point $z \in D^{n}$, we define a unitary operator $U_{z}$ on $L^{2}\left(D^{n}\right)$ by

$$
U_{z} h(w)=h \circ \phi_{z}(w) k_{z}(w)
$$

for all $h \in L^{2}\left(D^{n}\right)$. Thus $U_{z}^{*} T_{f} U_{z}=T_{f \circ \phi_{z}}$. If $T_{f}$ essentially commutes with $T_{g}$, then $T_{f \circ\left(z_{1}, 0, \ldots, 0\right)}$ essentially commutes with $T_{g \circ \phi_{\left(z_{1}, 0, \ldots, 0\right)}}$.

Replacing $f$ and $g$ respectively by $f \circ \phi_{\left(z_{1}, 0, \ldots, 0\right)}$ and $g \circ \phi_{\left(z_{1}, 0, \ldots, 0\right)}$ in the above argument, we can get that for all $z_{1}$ in $D$,

$$
\frac{\partial\left(f \circ \phi_{\left(z_{1}, 0, \ldots, 0\right)}\right)}{\partial z_{1}}\left(0, z_{2}, \ldots, z_{n}\right) \frac{\partial\left(g \circ \phi_{\left(z_{1}, 0, \ldots, 0\right)}\right)}{\partial z_{1}}\left(0, z_{2}, \ldots, z_{n}\right)=0
$$

on $T^{n-1}$. But

$$
\frac{\partial\left(f \circ \phi_{\left(z_{1}, 0, \ldots, 0\right)}\right)}{\partial z_{1}}\left(0, z_{2}, \ldots, z_{n}\right)=\left(\left|z_{1}\right|^{2}-1\right) \frac{\partial f}{\partial z_{1}}\left(z_{1}, z_{2}, \ldots, z_{n}\right) .
$$


This implies

$$
\frac{\partial f}{\partial z_{1}}\left(z_{1}, z_{2}, \ldots, z_{n}\right) \frac{\partial g}{\partial z_{1}}\left(z_{1}, z_{2}, \ldots, z_{n}\right)=0
$$

for $\left(z_{2}, \ldots, z_{n}\right) \in T^{n-1}$ and $z_{1} \in D$. Thus either

$$
\frac{\partial f}{\partial z_{1}}\left(z_{1}, z_{2}, \ldots, z_{n}\right) \quad \text { or } \quad \frac{\partial g}{\partial z_{1}}\left(z_{1}, z_{2}, \ldots, z_{n}\right)
$$

is zero on $D^{n}$. This completes the proof of the Theorem.

\section{ACKNOWLEDGMENT}

The authors are grateful to the referee for useful suggestions.

\section{REFERENCES}

[AFR] P. Ahern, M. Flores and W. Rudin, An invariant volume-mean-value property, J. Funct. Anal. 111 (1993), 380-397. MR 94b:31002

[AG] S. Axler and P. Gorkin, Algebras on the disk and doubly commuting operators, Trans. Amer. Math. Soc. 309 (1988), 711-723. MR 90a:46133

[R] W. Rudin, Function theory on the polydiscs, Benjamin, New York, 1969. MR 41:501

[SW] E. M. Stein and G. Weiss, Introduction to Fourier analysis on euclidean spaces, Princeton Univ. Press, 1971. MR 46:4102

[Z] D. Zheng, Hankel operators and Toeplitz operators on the Bergman space, J. Funct. Anal. 83 (1989), 98-120. MR 91b:47057

Department of Mathematics, Sichuan University, Chengdu, People's Republic of CHINA

Department of Mathematics, Michigan State University, East Lansing, Michigan 48824

Current address: Department of Mathematics, Vanderbilt University, Nashville, Tennessee 37240 